\title{
La raison du plus fort est toujours la meilleure
}

Diesen Satz aus der Fabel von La Fontaine kennen wir wohl alle seit unserer Gymnasialzeit! Ich habe Lust, ihn heute auf die Tabakindustrie anzuwenden: Sie hat angekündigt, dass sie ihre Preise senken wird, weil der Verkauf von Zigaretten um $10 \%$ eingebrochen sei.

Die Reaktion des BAG, man werde möglichst bald die Tabakbesteuerung heraufsetzen, folgte zwar sogleich - aber was wird der nächste Streich sein?

Es ist wichtig, dass wir uns bewusst sind, dass die Schweiz von aussen in Sachen Tabakbesteuerung und wenig eingeschränkter Werbung für Zigaretten in Europa keineswegs als beispielhaft angesehen wird. Das hängt nicht zuletzt damit zusammen, dass mehrere Tabakmultis ihren Sitz in unserem Land haben und demnach von seiten der Politikerinnen und Politiker gerne pfleglich behandelt werden.

Das Vorgehen der Zigarettenindustrie entspricht natürlich auch dem Prinzip, dass Situationsveränderungen wesentlich leichter zu er- reichen sind als Verhaltensänderungen. Wie können wir Ärztinnen und Ärzte solch vereinfachendem Denken erfolgreich entgegentreten? Alles redet von Prävention. Solche schier primitiven Prinzipien sind natürlich massenwirksamer als Einwirken zugunsten von Empowerment!

Wir Ärztinnen und Ärzte wollen auch in Zukunft Menschen mit einem gut entwickelten Bewusstsein für Verantwortung der Gemeinschaft und sich selbst gegenüber; Menschen, die Verlockungen des Augenblicks als beliebig und nicht zwingend erkennen und mit Verzicht und Frustrationen umgehen können. Sonst ist es in Zukunft um die Volksgesundheit schlecht bestellt. Aus diesem Grund setzen wir uns weiterhin für ein sinnvolles Ermächtigen Empowerment eben - jedes einzelnen ein.

Dr. med. Ursula Steiner-König, Mitglied des Zentralvorstandes der FMH, Ressort Prävention und Gesundheit 